\title{
Enseignement en Eco-Conception : une expérience à l'ENS de Cachan
}

\author{
Vincent DEBUSSCHERE ${ }^{(1)}$, Bernard MULTON ${ }^{(1)}$, Hamid BEN AHMED $^{(1)}$, Catherine RICORDEL $^{(2)}$, Marie- \\ Dominique de CAYEUX ${ }^{(2)}$, Philippe GOURRONC ${ }^{(2)}$, Yannick LEGUERN ${ }^{(3)}$ \\ ${ }^{(1)}$ nom.prenom@bretagne.ens-cachan.fr ${ }^{(2)}$ catherinericordel@ecole-eme.com ${ }^{(3)}$ yannick.leguern@biois.com \\ ${ }^{(1)}$ ENS de Cachan site de Bretagne - Campus de Ker Lann - Avenue R. Schuman - 35170 BRUZ \\ ${ }^{(2)}$ Ecole des Métiers de l'Environnement $-{ }^{(3)}$ Bio Intelligence Services
}

RESUME : L'éco-conception est une démarche de conception s'inscrivant clairement dans la logique du développement durable, elle concerne aussi bien les services que les dispositifs matériels. Dans le cadre d'une formation en Mécatronique sur le site de Bretagne de l'ENS de Cachan, nous avons mis en place depuis la rentrée 2007, une unité d'enseignement (UE) en éco-conception au niveau M1, notamment grâce à l'appui de l'Ecole des Métiers de l'Environnement qui disposait déjà d'un savoir-faire et d'un réseau de contacts. Les enseignements en éco-conception vont être, sans aucun doute, amenés à se généraliser et nous avons souhaité, à travers ce papier, faire profiter de notre courte expérience ( 2 ans à la date de soumission du papier). Après une première année expérimentale, cette UE comprend trois parties, la première est constituée de 4 séances de cours magistraux destinés à donner ce que nous avons estimé être des fondamentaux pluridisciplinaires (4 enseignants concernés : SVT, chimie et Génie Electrique). La deuxième partie est assurée par un intervenant d'une entreprise privée pionnière dans cette thématique (Bio Intelligence Service) qui possède une très bonne expérience en Analyses du Cycle de Vie (ACV) et qui offre une vision concrète du domaine. Enfin, la dernière partie est une séance de travaux pratiques de réalisation d'une ACV comparative sur un logiciel commercial (en l'occurrence EIME, mais d'autres sont également possibles). Le papier résume les contenus de ces trois parties ainsi que les modalités d'évaluation mises en place.

Mots clés : Eco-conception, mécatronique, enseignement pluridisciplinaire.

\section{INTRODUCTION}

L'éco-conception est une démarche de conception qui consiste à considérer l'ensemble du cycle de vie du produit étudié ainsi qu'un ensemble d'impacts environnementaux de natures variées (matières premières non renouvelables, gaz à effet de serre, eutrophisation de l'eau...) et par essence nécessitant des connaissances pluridisciplinaires. Il s'agit ainsi de faire en sorte que le produit éco-conçu conduise à des impacts minimisés. Notons qu'il est souvent difficile voire impossible de minimiser simultanément tous les impacts, dans ce cas, il faut définir un compromis (par pondération des impacts) ou encore faire des choix. L'éco-conception constitue donc l'un des fondements du développement durable, c'est la raison pour laquelle elle devra progressivement pénétrer tous les programmes d'enseignement supérieur. A la base de cette démarche se trouve l'analyse du cycle de vie (ACV) sur laquelle on commence à trouver de bons ouvrages [1]. L'Ecoconception a d'abord commencé à être utilisée dans les domaines du génie des procédés où des process industriels très polluants étaient utilisés, ses applications s'étendent aujourd'hui vers l'ensemble des sciences pour l'ingénieur et notamment le génie électrique [2]. Son enseignement se diffuse progressivement et nous avons souhaité l'inclure dans le programme de la formation en mécatronique que nous avons démarrée sur le site de Bretagne de l'ENS de Cachan en 2006 [3] et dont l'objectif était de former de futurs enseignants et/ou chercheurs dans les domaines de l'électricité, de la mécanique et de la mécatronique. La formation, destinée à des élèves normaliens ou non, est fondée sur la préparation simultanée de deux licences et deux mas- ters (en «électronique » et en «mécanique») de l'Université de Rennes 1 associée à des unités d'enseignement spécifiques en mécatronique, dont celle d'éco-conception située au premier semestre du niveau M1 pour des raisons de commodité. Cette unité aurait très bien pu être insérée dans le programme de L3 et cela aurait même sans doute été souhaitable de façon à sensibiliser au plus tôt les étudiants à ces concepts. Cette UE compte pour 4 ECTS dans cette formation dont l'année de M1 totalise 85 ECTS. Nous proposons, dans ce papier, de décrire, en trois chapitres, les trois parties constituant ce cours ainsi que les modalités de contrôle des connaissances. La première partie de l'UE est composée de quatre séances de cours magistraux sur ce que nous avons considéré constituer des fondamentaux. Notamment en ce qui concerne les impacts environnementaux, nous souhaitions que nos étudiants comprennent au mieux leur sens du moins pour quelques uns car il est impossible à l'échelle d'une telle UE de tous les analyser. D'une façon générale pour l'ensemble des informations transmises, nous souhaitons qu'ils acquièrent l'esprit critique nécessaire dans ce domaine en pleine émergence et qui aura sans doute encore à subir des évolutions.

\section{ENSEIGNEMENTS FONDAMENTAUX}

\subsection{Introduction à l'éco-conception}

Il s'agit ici d'introduire, après les fondements de l'écoconception le principe de la méthodologie d'analyse de cycle de vie, et la normalisation associée (série des normes ISO 14000). Les différentes étapes du cycle de vie d'un produit (fig. 1) ainsi que les différentes catégories d'impacts environnementaux sont décrits. Les flux 
entrants (matières premières, énergie, eau) et sortants (déchets, émissions liquides et gazeuses) conduisent à des impacts environnementaux et l'on met en évidence la nécessité, pour optimiser un produit ou service, d'étudier l'ensemble de son cycle de vie pour éviter les transferts de pollution d'une phase à l'autre.

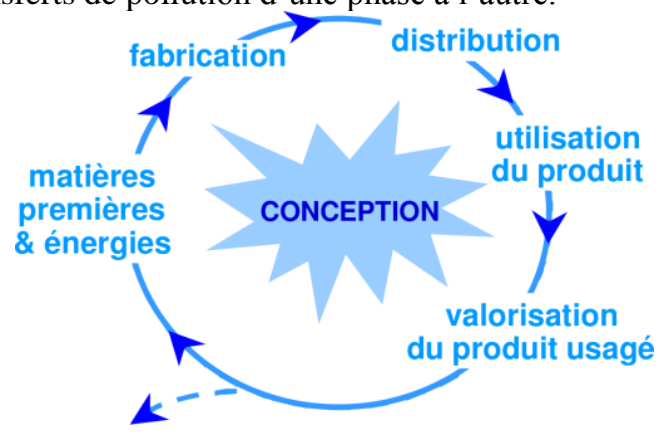

fig 1 : Cycle de vie d'un produit

Les démarches peuvent être exhaustives (cas de l'ACV où tous les impacts sont évalués sur toutes les étapes du cycle de vie) ou sélectives comme par exemple l'évaluation du seul «contenu énergétique» sur l'ensemble du cycle de vie ou encore l'Evaluation Simplifiée et Qualitative du Cycle de Vie (ESQCV) [4,5] qui n'est employée que pour améliorer un produit mais pas pour le comparer à d'autres.

Les quatre étapes de l'ACV sont ensuite détaillées, conformément à la norme ISO 14040 (fig 2).

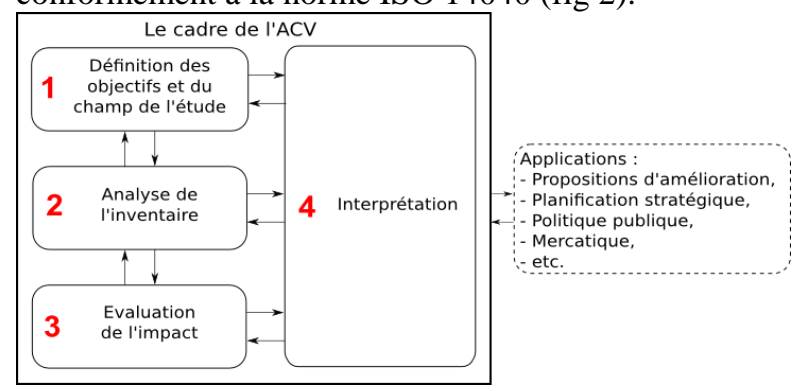

fig 2 : Les étapes de l'ACV [AFNOR]

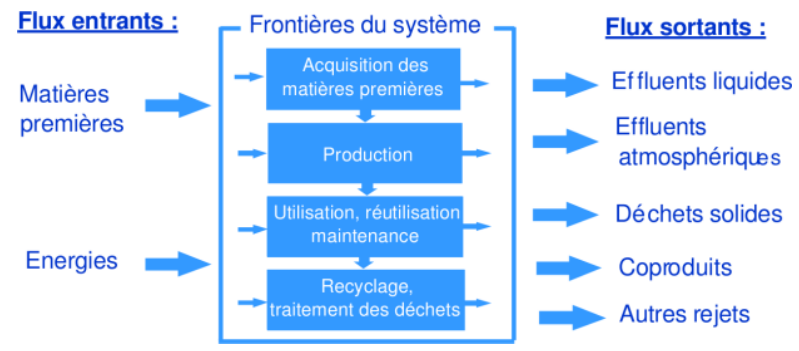

fig 3 : Inventaire du cycle de vie : première phase de l'ACV d'un produit [AFNOR]

L'étape 1 correspond à la définition des objectifs et du champ de l'étude. Il s'agit notamment de présenter l'objectif visé en termes de produits ou gammes de produits à comparer, de fonction étudiée et d'application de référence. Les limites du domaine à analyser sont dressées en fonction de l'objectif.

L'étape 2 dite inventaire ou écobilan consiste à recueillir les données d'entrée et de sortie aux frontières du système à partir d'un mode de collecte de données préalablement défini (fig 3). La qualité des données doit faire l'objet d'exigences particulières car elle conditionne la fiabilité des résultats de l'étude.

L'étape 3, évaluation des impacts sur l'environnement, consiste à rassembler les données recueillies en fonction des impacts qu'elles génèrent. Un jeu d'indicateurs proposé dans la norme iso 14040 permet alors de classifier les impacts.

La dernière étape consiste à interpréter les résultats de manière à cibler les améliorations potentielles pour augmenter la performance environnementale du système étudié en fonction du contexte.

\subsection{Compréhension des impacts environnementaux}

Les impacts environnementaux sont très nombreux, ils sont très variés et ont des conséquences extrêmement différentes allant de l'épuisement des ressources à l'action sur la santé humaine en passant par l'accroissement de l'effet de serre terrestre. Ils font appels à des connaissances pluridisciplinaires et sont généralement agrégés pour simplifier l'analyse ce qui nécessite de les pondérer. Il nous semble indispensable de conserver un esprit critique suffisant pour interpréter au mieux les résultats.

Les impacts environnementaux peuvent être classés en 5 catégories :

- épuisement des ressources naturelles (unité : masse)

- rejets gazeux dans l'air (unité : masse)

- rejets dans l'eau (unité : masse)

- consommation d'eau (unité : volume)

- énergie renouvelable ou non (unité : joules)

Leurs agrégations peuvent être effectuées de différentes façons. La norme ISO14040 donne une liste d'indicateurs qui sont définis par des groupes d'experts au niveau international et donc admis par la communauté internationale et repris partiellement ou dans leur totalité dans chaque analyse de cycle de vie. A titre d'exemple, dans le logiciel EIME, dédié aux produits électriques et électroniques [6], les impacts suivants sont considérés :

- RMD (Raw Material Depletion) : L'épuisement des ressources naturelles estime le rapport entre les quantités de matières premières utilisées (charbon, gaz, pétrole, fer, aluminium, etc.) sur l'ensemble du cycle de vie du produit considéré et les rapporte aux quantités disponibles et exploitables par l'homme.

- ED (Energy Depletion) : La consommation d'énergie sur l'ensemble du cycle de vie de produit, incluant la puissance consommée en fonctionnement, à préciser lors de la modélisation du produit.

- WD (Water Depletion) : L'appauvrissement des ressources en eau correspond à la consommation d'eau sur l'ensemble du cycle de vie.

- GWP (Global Warning Potential) : La contribution à l'effet de serre comptabilise, en équivalent $\mathrm{CO} 2$, les émissions de gaz ayant un impact direct ou indirect sur l'effet de serre (NO2, CH4, CFC, etc.). 
- OD (Ozone Depletion): La contribution à l'appauvrissement de la couche d'ozone comptabilise, en équivalent CFC-1, les émissions de gaz ayant un effet direct ou indirect sur la couche d'ozone (CFC, halons, etc.).

- AT (Air Toxicity) : La contribution à la toxicité de l'air comptabilise les volumes critiques émis vers l'air de substances appartenant aux listes de substances toxiques en usage dans la Communauté européenne.

- POC (Photochemical Ozone Creation) : La création d'ozone photochimique comptabilise, en équivalent $\mathrm{C} 2 \mathrm{H} 4$, les émissions vers l'air de substances ayant un effet direct ou indirect sur la création d'ozone troposphérique (toluènes, benzène, acétone, etc.).

- AA (Air Acidification) : La contribution aux pluies acides comptabilise, en équivalent d'ions $\mathrm{H}+$, les émissions vers l'air de substances transformées en substances acides (SO2, NO2, NO, HCI, etc.).

- WT (Water Toxicity) : La contribution à la toxicité de l'eau comptabilise les quantités émises vers l'eau de substances appartenant aux listes de substances toxiques en usage dans la Communauté européenne.

- WE (Water Eutrophication): La contribution à l'eutrophisation des eaux comptabilise, en équivalent d'ions PO3-4, les émissions vers l'eau de substances contribuant à l'eutrophisation des eaux.

- HWP (Hazardous Waste Production) : La production de déchets dangereux en fin de vie évalue la quantité de déchets produits et figurant sur les listes en usage dans la Communauté européenne.

Ce préambule est destiné à mettre en évidence la complexité de cet aspect de la problématique de l'écoconception.

Dans la courte séance de 4 heures consacrées aux impacts environnementaux et réalisée par un professeur de SVT, nous avons choisi de focaliser sur l'effet de serre, la couche d'ozone stratosphérique (rôle protecteur, atteinte par les polluants), la production d'ozone troposphérique (toxique), l'eutrophisation de l'eau d'origine anthropique (excès d'apport de nutriments), et enfin, la bioaccumulation et la bioconcentration des polluants dans la chaîne alimentaire et ses effets sur la santé humaine.

Les gaz à effet de serre (GES) sont très divers. Ils possèdent tous des molécules à plus de deux atomes, leur architecture moléculaire détermine leur capacité à absorber puis à restituer de l'énergie dans le domaine infra-rouge. La contribution d'un GES au forçage radiatif de ses propriétés radiatives et de sa stabilité, sa durée de vie dans l'atmosphère.

\begin{tabular}{lcccc}
\hline & $\begin{array}{c}\text { Durée de } \\
\text { vie estimée }\end{array}$ & à 20 ans & $\begin{array}{c}\text { PRG } \\
100 \text { ans }\end{array}$ & 500 ans \\
\hline $\mathrm{CO} 2$ & & 1 & 1 & 1 \\
\hline $\mathrm{CH} 4$ & 12 ans & 62 & 23 & 7 \\
\hline $\mathrm{N} 2 \mathrm{O}$ & 114 ans & 275 & 296 & 156 \\
\hline $\mathrm{CHF} 3$ & 260 ans & 9400 & 12000 & 10000 \\
\hline $\mathrm{SF} 6$ & 3200 ans & 15100 & 22200 & 32400 \\
\hline
\end{tabular}

Tabl. 1 : Pouvoir de réchauffement global des GES
Le pouvoir de réchauffement global (PRG) du $\mathrm{CO} 2$ est pris en référence, le tableau 1 montre le PRG de quelques autres GES relativement à celui du $\mathrm{CO} 2$ et sur des durées de 20, 100 et 500 ans.

De plus la contribution à l'effet de serre va prendre en compte la quantité de gaz présente dans l'atmosphère ou prévue selon les scénarios d'évolution... Le tableau 2 montre les contributions relatives à l'effet de serre dans la période 1980-1990 de quatre GES.

\begin{tabular}{cccc}
\hline $\mathrm{CO} 2$ & $\mathrm{CH} 4$ & $\mathrm{~N} 2 \mathrm{O}$ & $\mathrm{CFC} 11,12$ \\
\hline $55 \%$ & $15 \%$ & $4 \%$ & $19 \%$ \\
\hline
\end{tabular}

Tabl. 2 : Contribution à l'effet de serre dans la période 1980-1990

Ces quelques données illustrent la nécessité de préciser les critères de quantification des impacts environnementaux. L'impact de la vapeur d'eau n'a pas été évoqué ici.

\subsection{Energies, ressources, conversions et impacts}

Dans le cadre de la formation mécatronique dans laquelle s'inscrit cette unité d'enseignement, la conversion d'énergie constitue un volet important. En outre, le critère énergie sur cycle de vie en tant qu'impact environnemental pèse généralement lourd dans l'ACV d'un système de conversion ou de production d'énergie, notamment électrique. C'est pourquoi nous avons choisi de détailler ces aspects.

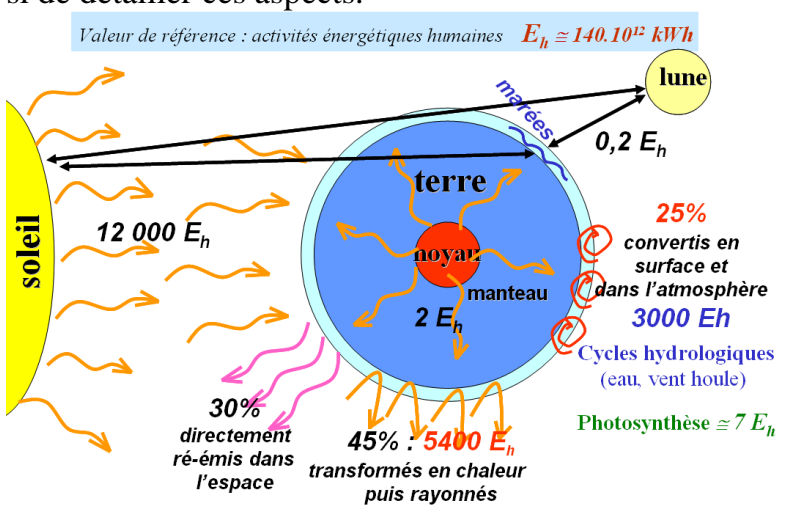

fig 4 : Diapositive des ressources renouvelables

Ce cours est ainsi destiné d'abord à prendre connaissance de la réalité des ressources énergétiques renouvelables et non renouvelables (fossiles et fissiles) de la planète terre. La problématique des ressources en matières premières non énergétiques est également abordée. La figure 4 montre la diapositive précisant les ressources annuelles renouvelables relativement à la consommation d'énergie primaire mondiale.

Ensuite, il s'agit de bien comprendre comment évoluent le rendement instantané en fonction du point de fonctionnement d'un convertisseur d'énergie, les différentes notions de rendements énergétiques, notamment sur 
cycles d'usage et sur cycle de vie ainsi que l'influence du point de fonctionnement :

Rendement en puissance ou instantané :

$$
\eta=\frac{P_{u}}{P_{a b s}}
$$

Rendement énergétique ou sur cycle d'usage :

$$
\eta=\frac{\int_{\text {cycle }}^{c y c l e} P_{u} \cdot d t}{P_{a b s} \cdot d t}=\frac{W_{u}}{W_{a b s}}
$$

Rendement sur cycle de vie (incluant donc l'énergie nécessaire à la fabrication et au recyclage) :

$$
\eta=\frac{W_{u}}{W_{\text {abs }}+W_{\text {fabrication+recyclage }}}
$$

Le cas des transports routiers sert de support à cette description des rendements. Les différentes visions du problème énergétique sont présentées qu'il s'agisse de l'amélioration des rendements de conversion, des carburants alternatifs (y compris l'électricité), du bilan « du puits à la roue » pour aller jusqu'à l'ACV du véhicule.

Les aspects économiques sont également analysés en faisant apparaître les différentes phases des coûts (investissement et fonctionnement) en particulier pour la production d'énergie électrique. Il apparaît que la durée de vie (durée d'amortissement dans le cas économique) intervient quasiment de la même façon dans une optimisation économique que dans une optimisation avec des critères environnementaux sur cycle de vie.

Enfin, la séance se termine par un panorama des différentes solutions de production d'électricité. Leurs impacts en termes d'émissions de gaz à effet de serre $(\mathrm{kg}$ eq $\mathrm{CO}_{2} / \mathrm{kWh}_{\mathrm{e}}$ ) sont comparés de façon critique.

\subsection{Cycle de vie des matériaux}

Cette séance, également de 4 heures, est destinée à bien comprendre l'origine des principaux impacts environnementaux d'un matériau. Elle est sous la responsabilité d'une chimiste qui connaît bien les procédés. Compte tenu de la faible durée de la séance, nous avons retenu deux matériaux : l'aluminium et le verre et, dans une moindre mesure, les composites.

Ce cours s'appuie sur le cours « matériaux » dispensé à l'EME. Il permet d'illustrer la méthodologie sur des exemples concrets qui pourront être directement utiles aux élèves. Différents aspects rencontrés en ACV sont présentés comme la prise en compte du recyclage ou des co-produits. A chaque étape du cycle de vie du matériau, du berceau à la tombe, les entrants, les sortants et les impacts environnementaux associés sont identifiés. La fabrication de verre et d'aluminium sont des activités à haute température, demandant une forte énergie, ce qui provoque l'émission de produits de combustion et de l'oxydation à haute température : on met en évidence les principaux problèmes rencontrés par ces industries en matière environnementale, en l'occurrence les émissions atmosphériques. Par ailleurs, la préparation du minerai pour la fabrication de l'aluminium crée des co-produits: les boues rouges. Leur filière de traitement est présentée au même titre que toutes les autres filières de traitement des autres co produits (poussières, crasses....)

Le bénéfice du recyclage de l'aluminium et du verre est détaillé. La monétarisation des impacts environnementaux liés au recyclage est présentée pour mettre en parallèle que les bénéfices du recyclage justifient les surcoûts à supporter par la société.

\section{ETUDES DE CAS- PRATIQUE D'UNE ACV}

Cette partie du cours est traitée par un intervenant d'une société spécialisée en $\mathrm{ACV}, B I O$ Intelligence Service.

Ce cours se déroule sur deux journées complètes permettant aux étudiants de connaitre et comprendre la méthode des analyses de cycle de vie et de la mettre en pratique.

La première journée est dédiée aux apports théoriques. Elle permet de comprendre comment et pourquoi est apparue nécessaire de développer une approche holistique permettant d'appréhender l'ensemble des enjeux environnementaux d'un produit sur son cycle de vie. Les principaux types d'utilisateurs et d'utilisation sont également présentés.

Un exemple de la méthode est présenté à travers la restitution d'une étude réalisée sur la filière bois énergie en comparaison avec les énergies conventionnelles utilisées pour des applications industrielles et notamment le séchage des sciages dans l'industrie du bois.

Dans un second temps, les référentiels normatifs (ISO 14040 et 14044) sont présentés et expliqués aux étudiants selon la chronologie classique de réalisation d'une étude ACV, cette fois dans le contexte d'une entreprise. Cette première journée conclut sur les développements méthodologiques en cours et sur les applications futures de l'ACV.

La seconde journée consiste en la réalisation d'une $\mathrm{ACV}$ basée sur un cas industriel concret. Elle consiste à comparer deux types d'emballages alimentaires selon différents critères environnementaux. Pour cela, les étudiants sont amenés à réaliser l'ACV selon les étapes décrites lors de la première journée. Ils doivent dans un premier temps décrire le système à étudier et proposer l'unité fonctionnelle permettant de comparer les résultats des deux types d'emballages de façon cohérente. Ensuite, un jeu de données leur est fourni et les étudiants doivent réaliser les calculs dans un fichier Excel, ce qui constitue l'originalité majeure en comparaison de l'utilisation d'un logiciel ACV (partie 4). En effet, ils doivent manipuler directement des inventaires de cycle de vie et intégrer les formules de calculs permettant de calculer une liste restreinte d'indicateurs proposés par l'enseignant. L'intérêt d'une manipulation de ce jeu de données sous Excel est de comprendre les fondements du fonctionnement des logiciels disponibles 
dans le commerce et de cerner l'ensemble des enjeux méthodologiques relatifs à ce type d'étude.

\section{SEANCE DE TP SUR UN LOGICIEL}

L'objectif de ce TP est de présenter une méthode d'analyse sur cycle de vie fondée sur l'utilisation d'un logiciel spécialisé dans la gestion de bases de données d'inventaires sur cycle de vie.

Lors d'une ACV, l'agrégation de l'ensemble des données d'impacts environnementaux peut représenter une partie non négligeable de l'étude mais ne présente que peu d'intérêts en termes méthodologiques. Il existe ainsi plusieurs types de logiciels dont le but est d'aider à la réalisation de cette étape de l'analyse sur cycle de vie. Les premiers proposent des évaluations qualitatives, comme des indices écologiques, les seconds proposent des évaluations sous forme de check-lists spécialisées dans des domaines précis et enfin les derniers proposent des évaluations quantitatives, nécessitant une grande quantité de données mises à jour régulièrement. Dans cette dernière catégorie, certains logiciels sont dédiés uniquement à des experts en gestion environnementale (GABI, PE en Allemagne [7] ou TEAM, Ecobilan en France [8]) et d'autre prennent le parti d'être utilisables par des concepteurs non spécialistes des impacts environnementaux. Ils utilisent donc une double interface, la première servant à modéliser les produits et la seconde à gérer les données d'inventaires sur cycle de vie. Dans cette catégorie de logiciels nous pouvons citer Simapro, Pre-Consultant aux Pays-Bas [9] et EIME [6] de la société CODDE en France.

Ce TP exploite le logiciel EIME mais pourrait tout à fait être reproduit sur n'importe quel logiciel de ce type. L'utilisation de ce logiciel en tant qu'outil d'aide à la conception écologique de produits sur des cas simples a pour but de se familiariser avec son interface concepteur, et non expert en gestion environnementale. Au cours du TP les élèves ont accès à cette deuxième interface mais n'ont pas d'action sur les bases de données. Le logiciel EIME est élaboré autour d'une architecture de type client-serveur, afin de faciliter le partage en réseau de la base de données ainsi que des projets. Notons que les bases de données de la version éducation de EIME ne sont pas remises à jour. Elles contiennent de plus une version des bases de données plus ancienne que celle de la version industrielle à la date de l'achat (la version industrielle est remise à jour tout les ans à deux ans). L'exploitation des résultats reste donc plus qualitative que quantitative. Les principales étapes d'utilisation du logiciel se résument comme suit :

1. Description du produit par l'intermédiaire d'une liste de composants, matériaux ou process. Il est aussi possible de décrire les liaisons entre les différents composants du produit modélisé (voir fig 5).

2. Des messages (mis en place par l'expert environnemental) donnent à l'utilisateur des informations relatives à la réglementation ou à des exigences du client ou même du chef de projet concernant le produit en cours de conception.

3. Lors de l'évaluation d'un produit, il peut être comparé à un ou plusieurs autres produits types afin de visualiser les points forts et faibles de chacun sur les différents critères d'impact environnementaux.

Le TP commence par une prise en main du logiciel sur un exemple simple, déjà étudié lors de l'étude de cas pratique (cf chapitre 3). L'intérêt est alors double. En effet, outre l'apprentissage du maniement de l'interface de modélisation, les élèves réalisent en un temps court le travail effectué en un TP complet à la séance précédente, le logiciel se chargeant du calcul d'agrégation des impacts environnementaux à leur place. L'intérêt d'une aide à la conception de systèmes complexes est alors nettement perceptible. En outre, la comparaison des résultats obtenus dans ces deux calculs met en lumière la sensibilité des résultats de l'agrégation des impacts environnementaux du produit à la précision de sa modélisation. Le principal avantage et le principal défaut des bases de données d'inventaires d'analyse sur cycle de vie sont donc soulignés dès le début du TP : une nette diminution voire une disparition du temps consacré à la collecte (ou la mesure) de données, leur agrégation et représentation, contrebalancée par une dépendance significative aux valeurs stockées dans les bases de données proposées par le logiciel. Au final les résultats sont obtenus plus rapidement, mais leur analyse et surtout leur justification prend plus de temps.

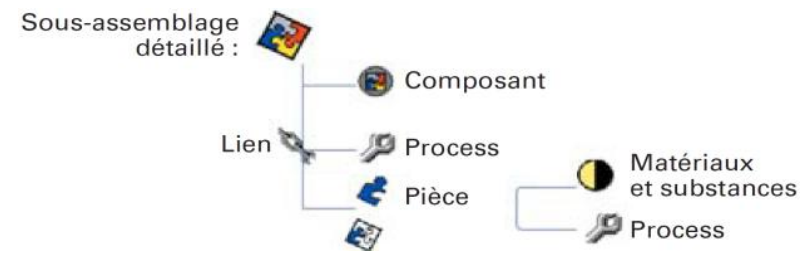

fig 5 : Modules à la disposition du concepteur dans le logiciel EIME

Suite à cette prise en main, l'objet principal du TP est la modélisation d'un bloc autonome d'éclairage de sécurité (BAES), objet consommateur d'énergie et, de notre point de vue, correspondant bien aux objectifs pédagogiques de notre formation. Cette foi-ci les élèves sont mis en position de concepteur, ayant à établir un cahier des charges réaliste de BAES à partir d'exemples tirés de documentations constructeurs [10]. Un BAES doit fournir une luminosité minimale en mode de défaillance (lorsque le courant est coupé) pendant un temps donné (typiquement quelque dizaines de lumens pendant une heure) et une luminosité de contrôle en mode de veille. Ce produit contient donc une batterie, une source lumineuse pour le mode de défaillance, une autre pour le mode de veille ainsi que divers constituants électriques (cartes de commande, recharge de la batterie) et plastiques (boitiers).

Suivant le choix de la source de luminosité en mode de défaillance et la durée du mode de défaillance, les bat- 
teries vont voir leur nombre de cellules varier ainsi que les impacts environnementaux du BAES. Dans cette version de logiciel, les élèves peuvent choisir différentes technologies d'éclairage comme celles à tube fluorescent, à LED et à incandescence (nous leur fournissons les différentes efficacités lumineuses) et d'accumulateurs Nickel-Cadmium et Lithium-Ion. Le choix du "packaging" et du mode de distribution est aussi discuté (livraison par palettes en grande surface, installation manuelle depuis le site de fabrication, etc.). La simulation du cycle de vie du composant est décomposée en trois étapes. La première consiste en sa modélisation, qui permet au logiciel de déterminer les impacts environnementaux des phases du cycle de vie autres que la phase utile et le transport (extraction des matières premières, production des sous-produits, fabrication, etc.). La seconde étape est la description détaillée de la phase utile du produit. Sont renseignées les consommations en veille et actives du produit, sa durée de vie. S'ajoutent à ces considérations l'étape d'installation du produit (incluant son emballage par exemple) et la phase de maintenance (incluant les composants qui vont nécessiter un remplacement pendant cette durée). Ces deux derniers points sont à compléter manuellement par le concepteur, le vieillissement des matériaux ou sous-composants n'étant pas proposé par le logiciel. La dernière étape est la fin de vie du produit. Dans cette version du logiciel, nous ne pouvons choisir qu'entre mise à dépôt et recyclage partiel (suivant la description du composant le logiciel estime que certaines parties pourront être démontées et réutilisées).

Notons que la seconde étape joue un rôle significatif dans nos comparaisons d'écobilans du fait que nous comparons des éclairages à incandescence, fluorescence ou LEDs, qui ne présentent pas les mêmes durées de vie. Il faut dès lors prévoir le remplacement de la lampe suivant la durée totale d'utilisation spécifiée dans l'étape descriptive de la phase de fonctionnement.

Le compte rendu du TP comprend les hypothèses d'analyse sur cycle de vie (notamment l'unité fonctionnelle et la frontières du champ d'étude, fondamentaux dans ce TP basé sur des comparaisons d'écobilans) la mise en place de la modélisation puis la comparaison des différentes solutions, avec graphiques et commentaires à l'appui. Nous présentons fig 6 un exemple de comparaison de deux écobilans, entre un BAES avec lampe à incandescence et un second avec des lampes fluorescentes. Le BAES dont l'éclairage de secours est effectué par des lampes fluorescentes est pris comme référent, et le second composant est exprimé en fonction du premier. Nous ne nous permettons pas de conclure quand à choix d'un BAES devant un autre avec l'âge et l'adéquation des données d'une part et la précision de la modélisation d'autre part. Cependant ce type de présentation permet visuellement de se faire une première idée.
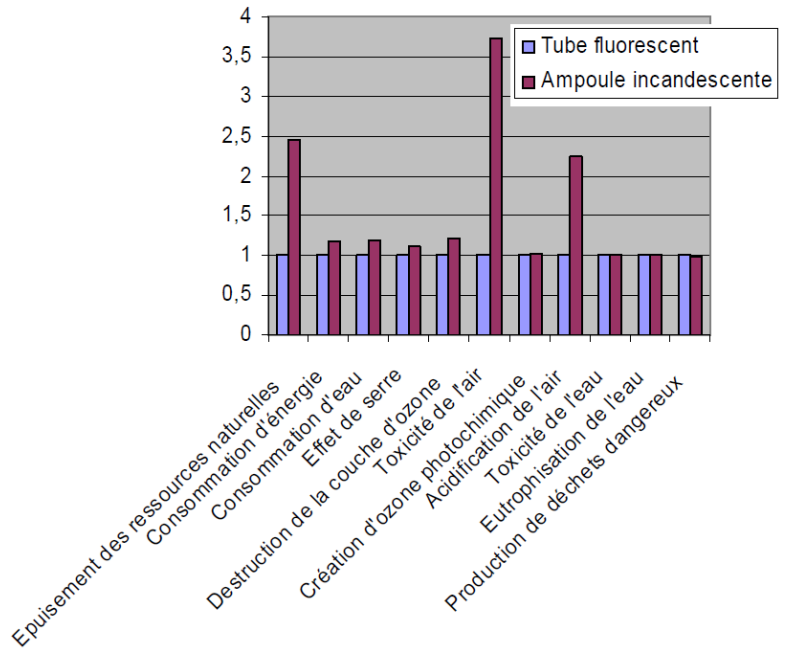

fig 6 : Comparaison de deux BAES, à fluorescence (référence) et incandescence

Les élèves ont globalement une grande latitude d'action sur la modélisation du produit. De ce fait chaque groupe de TP obtient des résultats différents, apportant des commentaires très instructifs pour de futurs concepteurs amenés à prendre en compte des critères aussi généraux que les impacts environnementaux.

\section{SYNTHESE - CONCLUSION}

Tout d'abord, nous proposons un tableau de synthèse des séances et volumes horaires associés dans la dernière version en date, sachant que cette UE est encore susceptible d'évoluer (pour progresser...) :

\begin{tabular}{|l|l|l|l|}
\hline Séances & Forme/heures & Evaluation & Enseignants \\
\hline fondamentaux & $4 \times 4$ cours & $\begin{array}{l}\text { Examen } \\
\text { écrit }+ \\
\text { exercice } \\
\text { libre noté }\end{array}$ & $\begin{array}{l}\text { 3 enseignants } \\
\text { (SPI, chimie, } \\
\text { SVT) }\end{array}$ \\
\hline $\begin{array}{l}\text { Etudes de cas. } \\
\text { Traitement } \\
\text { des données }\end{array}$ & $\begin{array}{l}2 \times 6 \mathrm{~h} \\
\text { cours }+\mathrm{TP} \\
\text { excel }\end{array}$ & TP noté & $\begin{array}{l}1 \text { intervenant } \\
\text { entreprise }\end{array}$ \\
\hline $\begin{array}{l}\text { Emploi logi- } \\
\text { ciel ACV }\end{array}$ & $\begin{array}{l}1 \mathrm{TP} \text { de } 4 \mathrm{~h} \\
\text { sur PC }\end{array}$ & TP noté & $\begin{array}{l}\text { 1 enseignant } \\
\text { SPI }\end{array}$ \\
\hline
\end{tabular}

Tabl. 3 : Synthèse des contenus de l'UE

L'enseignement de l'éco-conception nécessite une pluridisciplinarité assez importante. Dans le module d'initiation que nous avons construit, nous intégrons des cours magistraux en SPI (énergie), génie des procédés (vie des matériaux) et SVT (impacts environnementaux ainsi que des travaux pratiques allant jusqu'à la prise en main d'un logiciel spécialisé en ACV. L'évaluation des élèves, opération toujours délicate, est encore plus difficile qu'à l'accoutumée. Elle est ici réalisée par la synthèse d'un examen écrit d'évaluation des acquis des cours magistraux auquel s'ajoutent les notes des deux séances de TP et d'un exercice effectué en 
temps libre. L'examen écrit comporte autant de parties qu'il y a de séquences de cours magistraux différentes.

Les difficultés de réalisation d'une telle unité d'enseignement sont dues principalement au nombre élevé d'intervenants (à cause de la pluridisciplarité), impliquant de surcroit des personnes du milieu professionnel. En effet, dans notre cas, chaque séquence de cours magistral ainsi que chaque TP est assuré par une personne différente.

Notons que les séances de TP sont assurées jusqu'à présent sur le logiciel EIME, dont l'Ecole des Métiers de l'Environnement possède une licence éducation depuis maintenant quatre ans (4500 euros à l'époque pour 20 postes). Il est toutefois possible d'effectuer un TP similaire sur un logiciel gratuit, comme celui proposé par l'ADEME, Bilan Produit 2008. Celui-ci contient un extrait de la base de données EcoInvent (base de données d'ACV d'origine Suisse) et utilise le logiciel $E x$ cel. Nous pensons que le choix du logiciel doit plutôt être dicté par les objectifs de la formation et le niveau de spécialisation attendu. Pour une initiation, comme c'est ici le cas, un logiciel gratuit est amplement suffisant. S'il s'agit de former des techniciens ou des ingénieurs spécialistes, il est sans doute préférable d'employer un logiciel commercial du type EIME ou SIMAPRO qui leur offrira une première expérience sans doute utile lors de la recherche de stages et/ou d'emploi.

Assurée depuis trois ans, dans le cadre de la formation mécatronique du site de Bretagne de l'ENS Cachan, cette UE a subi des changements chaque année, mais leur ampleur va heureusement en décroissant. Le bilan est positif, aussi bien pour les élèves que pour les professeurs. En effet, ce module propose un point de vue actuel et réaliste sur des connaissances qui ne se retrouvent pas toujours dans le cursus scolaire habituel.

\section{Bibliographie}

[1] P. CRETTAZ, M. SAADÉ, O. JOLLIET, «Analyse du cycle de vie - Comprendre et réaliser un écobilan ", Presses Polytechniques et Universitaires Romandes, 2005.

[2] B. MULTON, H. BEN AHMED, V. DEBUSSCHERE, J. SEIGNEURBIEUX, M. RUELLAN, Y. THIAUX, «Ecoconception : de nouveaux critères de conception en électrotechnique pour le développement durable », REE $n^{\circ} 5$, mai 2008, pp89-98.

[3] ENS de Cachan, site de Bretagne, programme du magistère de Mécatronique : www.mecatronique.bretagne.ens-cachan.fr.

[4] J.P. VENTERE, « conception écologique des produits », Les Techniques de l'Ingénieur, G 6000, 1997.

[5] J.P. VENTERE, "La qualité écologique des produits. Des écobilans aux écolabels », Editions AFNOR et Sang de la terre, 1995

[6] L. LESCUYER, "EIME, un outil d'aide à la conception des produits", Les Techniques de l'Ingénieur, G 6100 , 2004.

[7] Logiciel d'analyse sur cycle de vie GABI, PE Consultant. http://www.gabi-software.com/index.php?id=1159, visité le 18 aout 2009
[8] Logiciel d'analyse sur cycle de vie TEAM, Ecobilan. https://www.ecobilan.com/fr_team.php, visité le 18 aout 2009.

[9] Logiciel d'analyse sur cycle de vie SimaPro, PreConsultant. http://www.pre.nl/, visité le 18 aout 2009.

[10] Site internet de Safetymatic, société distribuant, installant et vérifiant des blocs autonomes (BAES et BAEH) et des sources centrales d'éclairage de sécurité. http://www.safetymatic.fr/, visité le 18 aout 2009. 